\section{Experimental Questions:}

Orienting spatial attention using long-term memory activates the hippocampus, and the same dorsal frontoparietal brain networks as when attention is guided by transient spatial cues (Summerfield et al. 2006; Kastner \& Ungerleider, 2000; Chun, 2000). Previously, however, neural activity elicited by mnemonic cues has not been dissociated from activity elicited by targets. In this study we lengthened and varied cue-target stimulus onset asynchronies in a perceptual discrimination task with complex scenes to allow isolation of cue- and targetrelated processes in memory-guided orienting. Our key aims were:

1. To validate that the hippocampus and dorsal frontoparietal networks are activated by mnemonic cues.

2. To determine whether hippocampal and dorsal frontoparietal regions are engaged by mnemonic cues independently of target processing.

\section{Findings \& Conclusions:}

1. Mnemonic spatial cues facilitated perceptual discrimination of targets embedded in natural scenes.

2. Mnemonic cues engaged the hippocampus, and the same dorsal frontoparietal networks identified by a previous study using mnemonic cues to guide orienting of spatial attention (Summerfield et al., 2006).

3. No cue-dependent differences in hippocampal or dorsal frontoparietal activity were observed during the target phase of the tas

\section{References:}

Chun, M. M. (2000). Contextual cueing of visual attention. Trends in Cognitive
Sciences, $4(5), 170-178$. Kastner, S., \& Ungerleider, L. G. (2000). Mechanisms of visual
human cortex, Annual Review of Neuroscience, 23, 315-341.

Summerfield, J. J., Lepsien, J., Giteleman, D. R., Mesulam, M. M., \& Nobre, A. C. 49(6), 905-916
Methods:

Learning Task

Participants searched for and learned the location of targets (images of keys) in natural scenes $24-48$ hours before completing the orienting task. Two-thirds of the scenes presented contained targets.

Orienting Task (completed in fMRI scanner)

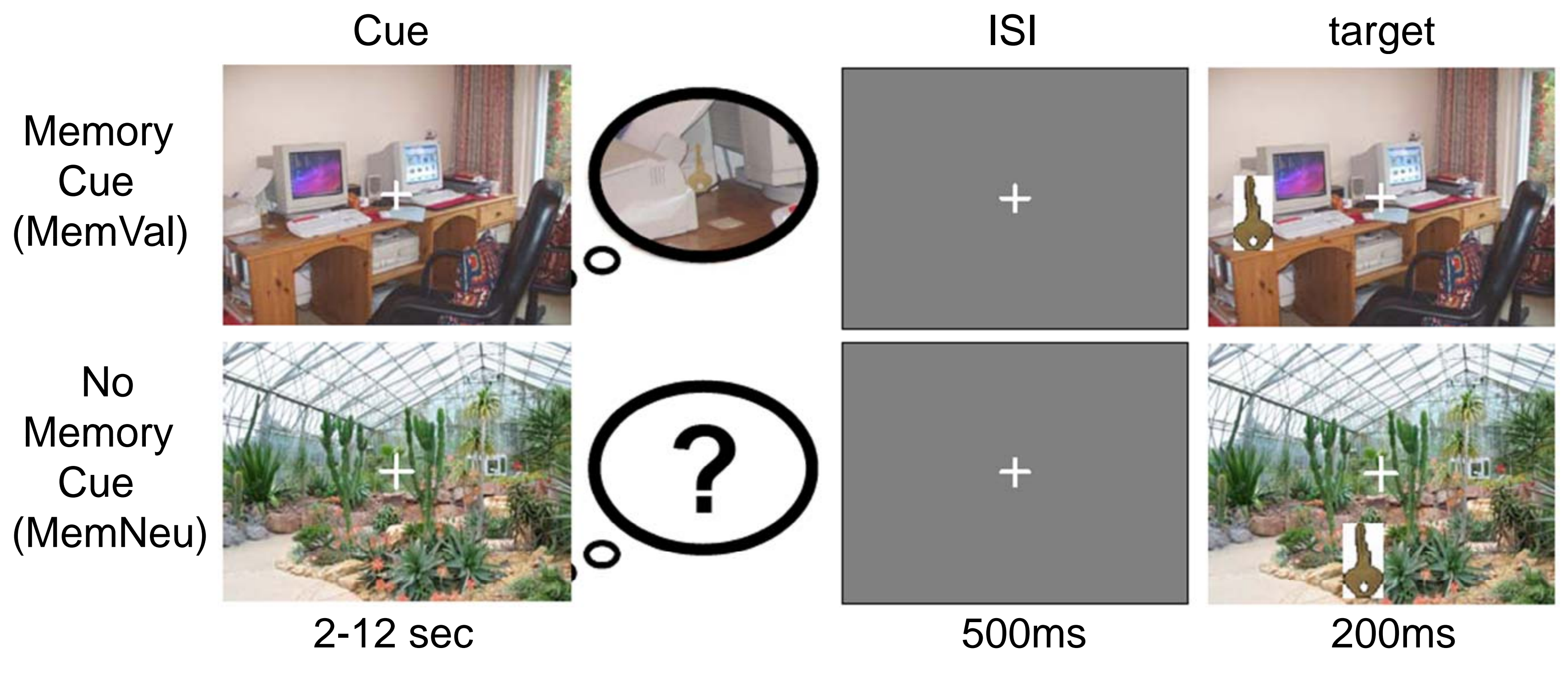

Mnemonic cues guided the orienting of attention.

No targets were present in cue scenes. Two-thirds of the cue scenes had contained targets in the learning task (MemVal condition). One-third of the cue scenes had not contained targets in the learning task (MemNeu condition). - Participants responded (yes/no) as to whether a target key was present. $50 \%$ of MemVal and $50 \%$ of MemNeu trials contained targets.

$\mathrm{N}=20$. Factors: cue (MemVal, MemNeu), target (present, absent).

fMRI Methods

3T Siemens TIM Trio, 12 channel headcoil

TR=3s, TE $=30 \mathrm{~ms}, 3 \mathrm{~mm}^{3}$ voxels, 45 axial slices

SPM5/MarsBar pre-processing and analysis, $7 \mathrm{~mm}$ smoothing

Events of interest: MemVal - MemNeu GLM analysis, FIR analysis.

\section{Behavioural Results:}
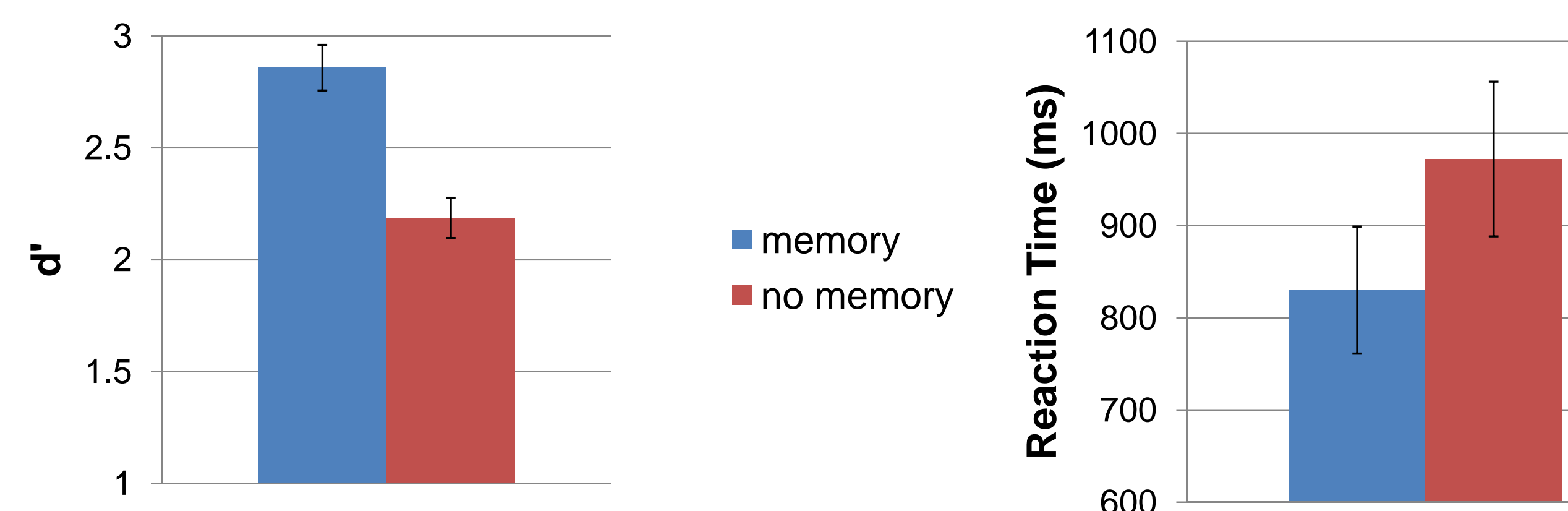

Long-term memory cues facilitated perceptual discrimination of targets (d'cuing effect: $(14)=4.06, p<001$ ) and reaction times (RT cuing effect: $t(14)=-3.14, p<01)$ in the orienting task.
fMRI Results:


Left Hippocampus
$[x y z=-30,-15,-18]$

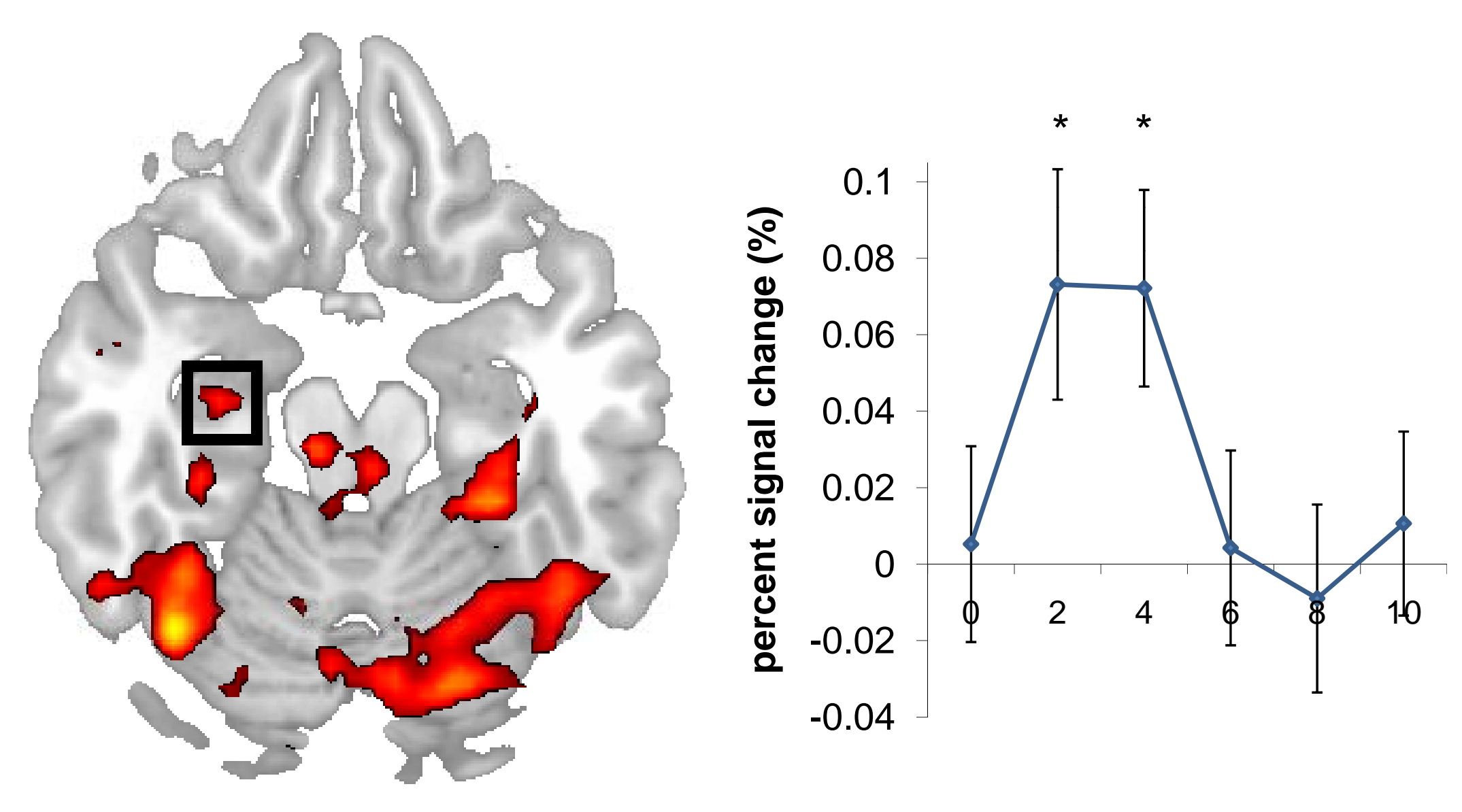

Memory cues engaged the same left hippocamal region observed by Summerfield et al (2006) significanty more thed no memory cues (MemVal - MemNeu comparison, P PDP<0.05). No cue-related differences were observed in the target phase (MemVal - MemNeu, all voxels $P_{F D P}>0.05$ ). FIR analysis of the time-course of hippocampal activity in the cue phase of MemVal trials is shown to the right $\left({ }^{*}=p<0.05\right)$

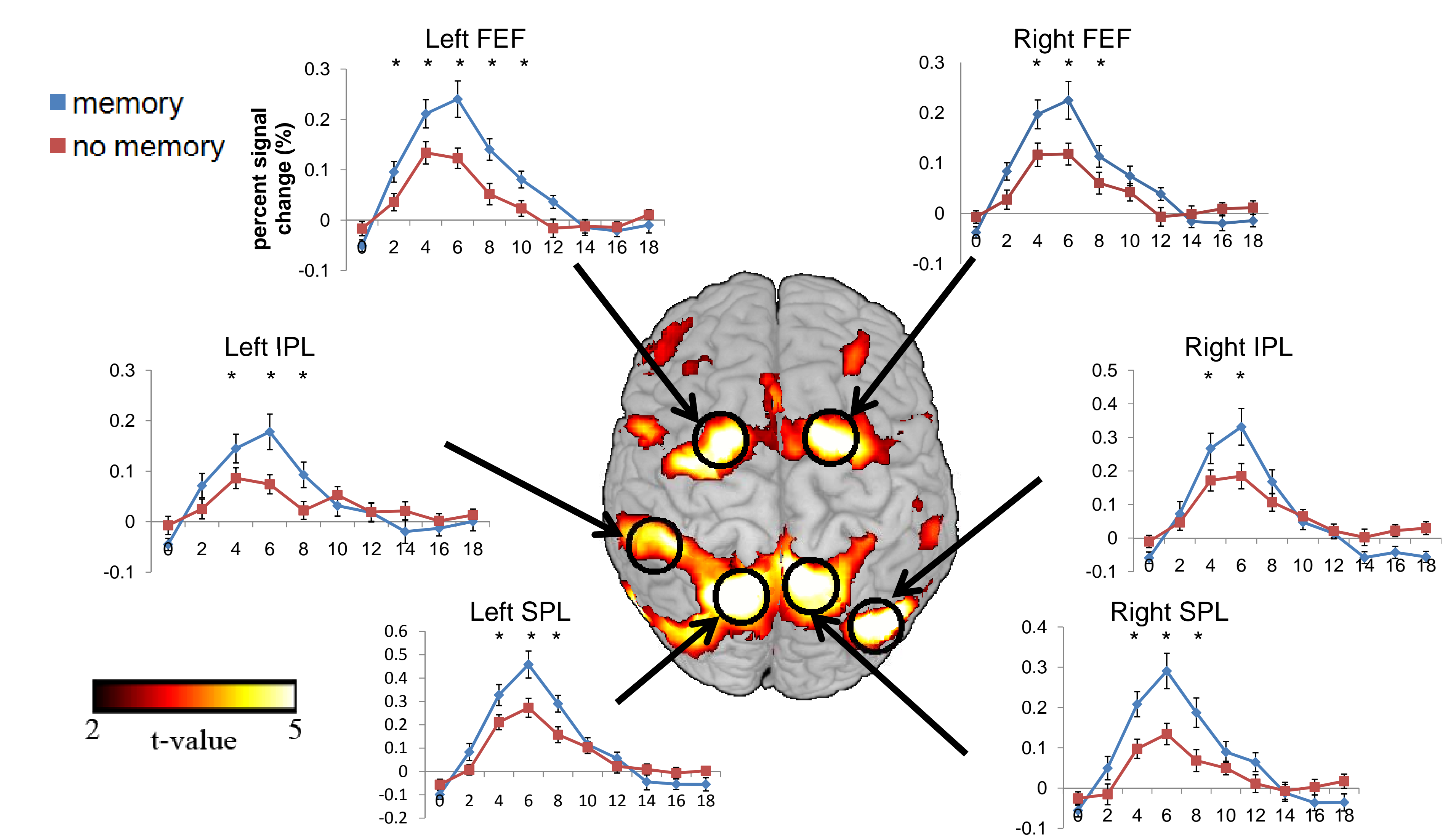

Whole brain analysis of the cue phase (shown in center) revealed greater dorsal frontoparietal orienting network engagement by memory cues than no memory cues (MemVal - MemNeu comparison, $\left.P_{F D R}<0.05\right)$. The outer plots show FIR analyses of peak activations $\left({ }^{*}=p<0.05\right)$. No cue-related differences were observed in the target phase (MemVal - MemNeu, all voxels PFDR $>0.05$ ).

\section{Notes:}

(please leave your email below to receive a pdf of this poster) 